\title{
An unusual case of nasopharyngial adenoid cystic carcinoma with intracranial and orbital extension: $A$ challenge for innovative conformal radiotherapy techniques
}

\author{
Touimi $\mathrm{SH}^{1,2 *}$, Seka E $\mathrm{E}^{1,2}$, Aarab $\mathrm{J}^{1,2}$, Omor $\mathrm{Y}^{2,3}$, Elkacemi $\mathrm{H}^{1,2}$, Elmajjaoui $\mathrm{S}^{1,2}$, Kebdani $\mathrm{T}^{1,2}$, Latib $\mathrm{R}^{2,3}$ and Benjaafar $\mathrm{N}^{1,2}$ \\ ${ }^{1}$ Department of Radiotherapy, National Institute of Oncology, Rabat, Morocco \\ ${ }^{2}$ Mohammed V University in Rabat, Morocco \\ ${ }^{3}$ Department of Radiology, National Institute of Oncology, Rabat, Morocco
}

\begin{abstract}
Adenoid cystic carcinoma of nasopharynx is rare. When tumor is resectable, surgery alone or followed by radiotherapy is frequently used for treatment. In the other hand, when tumor is not resectable, only radiotherapy can allow for a protracted local control obtaining. However, achievement of an optimal dose distribution is not always easy due to the location and the extent of the tumor. Therefore, very highly conformal radiotherapy techniques are needed. We present a rare and unresectable case of nasopharyngeal adenoid cystic carcinoma with intracranial and orbital extension that failed to be treated by photon volumetric arc therapy and highlight the need for proton or carbon ion therapy in the management of this entity.
\end{abstract}

\section{Introduction}

Adenoid cystic carcinoma accounts for only $5-10 \%$ of salivary gland tumors and less than $1 \%$ of all head and neck cancer [1]. It is characterized by slow relentless growth, extensive local invasion and a particular propensity for perineural spreading [2]. Its location in nasopharynx is very rare (approximately 0.13 to $0.48 \%$ of all malignant nasopharyngeal tumors) [3,4]. Surgical resection with or without radiotherapy is the mainstay of the management. However, in cases of advanced tumors where surgery is precluded because of the tumor extent, definitive radiotherapy remains the sole therapeutic option. When tumor attains or extends beyond the base of skull, the delivery of radiotherapy constitute a challenge. The need to spare some sensitive nearby subcortical structures of the brain render difficult the achievement of an optimal dose distribution. Indeed one have to deliver an effective, tumoricidal dose to the whole tumor while respecting the limits of tolerance of the anatomical and functional areas of major interest. Therefore, novel highly conformal radiation therapy techniques that have high therapeutic ratio are needed for the management of these tumors.

In this study, we report a rare case of nasopharyngeal adenoid cystic carcinoma with intracranial and orbital extension in a 20 year-old adolescent proposed for treatment by photon volumetric arc therapy (VMAT), and we discuss the role of innovative therapies in this entity.

\section{Case report}

She is a 20 years old patient with no significant medical history who consulted an ear, nose and neck doctor at "hôpital des spécialtés" of Rabat for a 12-month history of headaches and right earaches. The physical exam was normal for ear, nose and oral cavity. Fiberoptic scope of the nasopharynx showed a bulging mass of the nasopharynx that filled the right fossa of Rosenmuller. Biopsy specimen was taken for pathological study. Routine histological with hematoxylin and eosin staining (HES) and immunohistochemical (IHC) evaluation were performed. These concluded to an adenoid cystic carcinoma with cribriform and tubular patterns of nasopharynx. Head and neck MRI showed a tumor mass of the nasopharynx with intracranial and right orbit extension. The patient was referred to the National Institute of Oncology of Rabat for treatment. On examination, we noted a moderate right palpebral ptosis, but vision and ocular mobility are not impaired. Paresthesia and hypoesthesia of the right side of the face and limitation of the mouth opening were also found. Neck examination was normal for nodes. Chest and abdominal CT showed no distant metastasis. The tumor was classified T4N0M0. Surgery was not possible owing the tumor extension. Thus, definitive radiotherapy was choosed for treatment. We realized a volumetric arc therapy (VMAT) treatment planning with the goal of $70 \mathrm{~Gy}$ to the tumor. We made target volume and organs at risk delineation using MRI and CT images fusion. The patient abandoned her treatment after 10 courses of radiation therapy (Figures 1-3).

On Figure 3 showing statistics of dose-volume histogram, we can see that the limit of tolerance of the critical structures, which are optic chiasm, brainstem, optic nerve and temporal lobe, are not respected. Therefore, we referred her to the medical oncology department for palliative chemotherapy.

${ }^{\star}$ Correspondence to: Touimi SH, Department of Radiotherapy, National Institute of Oncology, Rabat, Morocco, E-mail: samiahajartouimi@gmail.com

Key words: adenoid cystic carcinoma, intracranial and orbital extension, radiotherapy, challenge

Received: March 29, 2020; Accepted: April 08, 2020; Published: April 14, 2020 
Touimi SH (2020) An unusual case of nasopharyngial adenoid cystic carcinoma with intracranial and orbital extension: A challenge for innovative conformal radiotherapy techniques

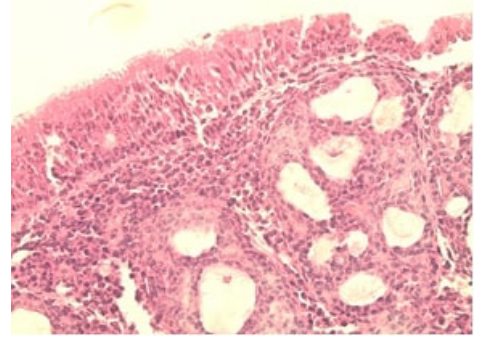

A



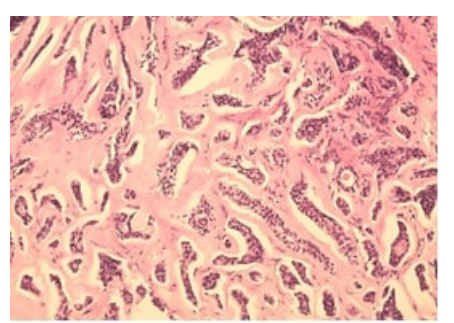

B



Figure 1. histologic and immunohistochemistry images. (A) HES: cribriform pattern (magnitude $\mathrm{x} 400$ ). (B) HES: tubular pattern (magnitude 400). (C) IHC: endoluminal cells positive for CK7 (magnitude 200). (D) IHC: myoepithelial cells positive for P63 (magnitude 400)

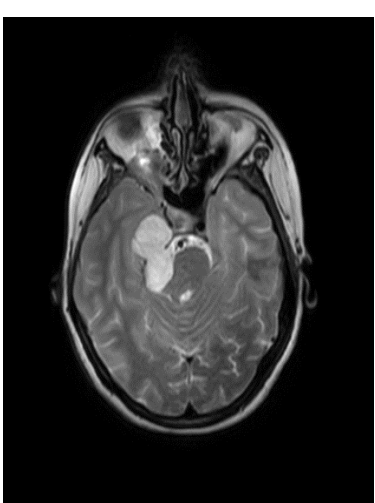

A

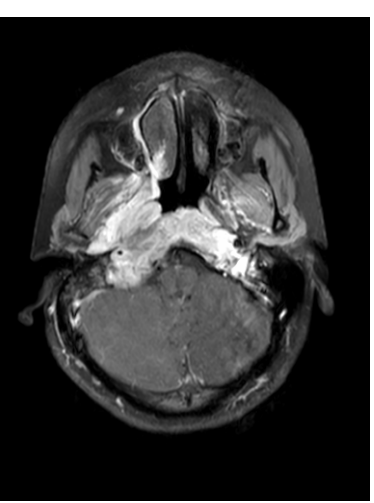

B



$\mathrm{C}$

Figure 2. Axial sectional MRI images showing nasopharyngeal tumor involving brain and right orbit. (A) axial T1 weighted sequence showing nasopharyngeal infiltrative tumor invading the parapharyngeal space. (B) axial T2 weighted sequence showing intracranial extension, tumor with close proximity to the brainstem and the right temporal lobe. (C) axial T1 weighted sequence showing intracranial and right orbit extension

\begin{tabular}{|c|c|c|c|c|c|c|c|c|}
\hline Structure & Volume $\left(\mathrm{cm}^{3}\right)$ & Min. Dose (Gy) & Max. Dose (Gy) & Mean Dose (Gy) & Hot Ref. (Gy) & Volume $>\left(\mathrm{cm}^{3}\right)$ & Volume $>(\%)$ & Conformity Index \\
\hline PTV 70 & 319.923 & 51.878 & 75.442 & 70.138 & 72.642 & 6.398 & 2.00 & 0.61 \\
\hline spinal cord & 20.685 & 0.264 & 39.478 & 23.167 & 33.787 & 0.414 & 2.00 & 0.00 \\
\hline brainstem & 28.395 & 29.722 & 64.169 & 49.544 & 60.770 & 0.568 & 2.00 & 0.01 \\
\hline Optique chiasm & 0.696 & 53.233 & 71.768 & 66.645 & 71.446 & 0.014 & 2.00 & 0.00 \\
\hline left optic nerve & 1.128 & 27.565 & 66.460 & 37.434 & 62.299 & 0.023 & 2.00 & 0.00 \\
\hline leftlens & 0.198 & 26.180 & 44.747 & 34.997 & 43.485 & 0.004 & 2.00 & \\
\hline left retina & 1.569 & 20.761 & 60.137 & 37.470 & 55.881 & 0.031 & 2.00 & \\
\hline left temporal lobe & 69.486 & 9.009 & 73.385 & 33.656 & 67.913 & 1.390 & 2.00 & \\
\hline
\end{tabular}

Figure 3. Image of table displaying DVH statistics 
Touimi SH (2020) An unusual case of nasopharyngial adenoid cystic carcinoma with intracranial and orbital extension: A challenge for innovative conformal radiotherapy techniques

\section{Discussion}

Adenoid cystic carcinoma is a rare salivary gland tumor that has been diagnosed exceptionally in the nasopharynx. Both histological study and immuno $\neg$ histochemical staining are very important for pathological diagnosis [4]. Because of its local aggressiveness and its slow growth, patients can be seen with bulky and unresectable tumor. In nasopharynx, adenoid cystic carcinoma tends to propagate following the cranial nerves tracts, causing very often an involvement of base of the skull and neural structures beyond. In literature, the rate of base of the skull invasion reaches $55 \%$ and the treatment strategy adopted was either surgery combined to post-operative radiotherapy or radiotherapy alone [1]. Cases with extension beyond the base of the skull and the subsequent therapeutic strategies have been exceptionally reported. These situations obviously constitute a challenge for radiotherapy because it is necessary to deliver a curative dose to the tumor without damaging the surrounding noble neural structures. Therefore, the radiation therapy techniques that offer a high therapeutic ratio are necessary. Volumetric arc therapy (VMAT) is available in our center, so we realized a subsequent treatment plan for our patient. However, the dosimetric study showed that the chiasm and the brainstem would receive unacceptable doses. Thus, arc therapy, although highly conformational is limited in this situation. However, it is not the technique itself that is limited but rather a problem inherent to photons. The dose deposited along the path of a photon beam is very often higher in the upstream healthy tissues it encounters than in the tumor itself. Moreover, because of the absence of defined course, the photon beam continues to irradiate tissues downstream at non-negligible dose. So a lot of the dose of a photon beam is deposited outside the target. This problem has been addressed by beam multiplication strategies and new conformational therapies such as IMRT (intensity modulated radiation therapy), tomotherapy and arc therapy [5]. However, with these means, the dose to healthy tissues is redistributed rather than reduced. In the literature, some studies have reported the superiority of neutron versus photon in adenoid cystic carcinoma [6,7]. Indeed neutrons have high linear energy transfer (LET) and significant relative biological effectiveness (RBE) for salivary gland tumors [8]. However, neutron therapy is subject to major late side effects due to the inability of neutrons to deliver the dose to tumor volume alone $[8,9]$. Therefore, they are not appropriate for treatment in this case where proximity to the brainstem, temporal lobe and chiasm requires maximal conformation. Unlike photons and neutrons, protons are charged particles. They have a straight course with a defined path length that depends on their kinetic energy. Depth-dose curve of protons shows a high peak (known as Bragg peak) at the end of the course where their relative biological effectiveness (RBE) is much higher. Therefore, the tissues located beyond are relatively protected. In addition, the dose deposited along the proton path is relatively low $[5,10]$. These physical advantages will allow for both maximum dose and greater biological efficiency in tumor while surrounding tissues receive low dose. Phan et al. had published a case of adenoid cystic carcinoma with intracranial involvement in a 71-year-old patient treated with proton therapy [11]. In their case, they made a comparison between IMRT treatment plan and IMPT (intensity modulated proton therapy) treatment plan. The advantage in dose distribution to target volume and organs at risk was in favor of proton therapy. Treatment with proton therapy have achieved local control for 2 years before relapse. The reported toxicities were dysgeusia (grade 2), mucositis (grade3) and dermatitis (grade 2). Other studies, such as those of Pommier et al. and Gentile et al. despite focused on cases with limited invasion of the base of the skull, have reported high control rates with proton therapy $(93 \%$ control at 5 years for the former and $100 \%$ local control for the latter) $[1,5]$. The acute and late toxicities observed were essentially grade 2 and 3 . Afani et al. reported a case of recurrent adenoid cystic carcinoma of the nasopharynx (after surgical resection and postoperative radiotherapy), invading the skull base and the meninges, treated with carbon ions [12]. This therapy resulted in an almost complete disappearance of the recurrence. The carbon ions have physical and radiobiological properties similar to those of protons but they are somewhat higher in dose distribution, with a tiny penumbra. Their therapeutic ratio is very high. Therefore, they offer great potential for the treatment of tumors near critical organ such as the brainstem and optic chiasm. In most studies, carbon ion therapy was used, with combination with photon IMRT, for a dose boost in macroscopic disease [12-15]. The results obtained have demonstrated its feasibility and its effectiveness in local and regional control of locally advanced adenoid cystic carcinoma of the head and neck.

These particulate therapies, proton therapy and carbon ion therapy are therefore the ones that brought greater effectiveness in terms of dose distribution and control of locally advanced inoperable adenoid cystic carcinomas of the nasopharynx. Our patient could benefit from it; but the cost and the availability of these treatments are a hindrance. On the other hand, though effective, long-term data are needed to evaluate late toxicities of these therapies compared to photon therapy.

\section{Conclusion}

Nasopharyngeal adenoid cystic carcinoma with intracranial and orbit extension is a very rarely described entity. Radiation therapy is the sole therapeutic option. New conformational photon therapies are not appropriate. Proton therapy and carbon ion therapy have shown encouraging results and should be proposed in this situation. Hence the interest in their development to enable their accessibility for patients who need them

\section{Acknowledgment}

We thank Dr. Mouna RIMANI of the laboratory « Centre d'Anatomie pathologique Hassan de Rabat " for histology and immunohistochemestry images.

\section{Disclosures}

The authors report no conflict of interest concerning the case in this paper.

\section{References}

1. Gentile MS, Yip D, Liebsch NJ (2017) Definitive proton beam therapy for adenoid cystic carcinoma of the nasopharynx involving the base of skull. Oral Oncology 65: 38-44. [Crossref]

2. Dong J, Tian L, Li S (2015) Differences in extension patterns between adenoid cystic carcinoma of the nasopharynx and nasopharyngeal carcinoma on MRI. Int J Clin Exp Pathol 8: 15960-15968. [Crossref]

3. Kuno H, Fujii S (2013) A case of adenoid cystic carcinoma arising from the nasopharynx. Jpn J Clin Oncol 43: 942

4. Liang YF, Kong B, Xiang WY (2014) Nasopharyngeal adenoid cystic carcinoma: A case report and review of the literature. Int J Clin Exp Pathol 7: 4516-4518.

5. Pommier P, Liebsch NJ, Deschler DG (2006) Proton beam radiation therapy for skull base adenoid cystic carcinoma. Arch Otolaryngol Head Neck Surg 132: 1242-1249.

6. Laramore GE, Krall JM, Griffin TW (1993) Neutron versus photon irradiation for unresectable salivary gland tumors: final report of an RTOG-MRC randomized clinical trial. Int J Radiat Oncol Biol Phys 27: 235-240.

7. Douglas JG, Laramore GE, Austin-Seymour M, Koh W, Stelzer K (2000) Treatmen of locally advanced adenoid cystic carcinoma of the head and neck with neutron radiotherapy. Int J Radiat Oncol Biol Phys 46: 551-557. 
Touimi SH (2020) An unusual case of nasopharyngial adenoid cystic carcinoma with intracranial and orbital extension: A challenge for innovative conformal radiotherapy techniques

8. Prott FJ, Micke O, Haverkamp U (2000) Results of fast neutron therapy of adenoid cystic carcinoma of the salivary glands. Anticancer Res 20: 3743-3750.

9. Schulz-Ertner D, Nikoghosyan A, Didinger B (2005) Therapy strategies for locally advanced adenoid cystic carcinomas using modern radiation therapy techniques. Cancer 104: 338-44.

10. Goitein M (2007) Radiation oncology: a physicist's-eye view. New York: Springer.

11. Phan J, Ng S, Pollard III C (2017) A rare case of unresectable adenoid cystic carcinoma of the nasopharynx treated with intensity modulated proton therapy. Cureus 9: e1688.

12. Afani L (2016) Carcinome adénoïde kystique du nasopharynx, une entité clinique rare en quête d'innovations thérapeutiques: cas clinique et revue de la littérature. Cancer Radiother.
13. Schulz-Ertner D, Nikoghosyan A, Jäkel O (2003) Feasibility and toxicity of combined photon and carbon ion radiotherapy for locally advanced adenoid cystic carcinomas. Int J Radiation Oncology Biol Phys 56: 91-398.

14. Jensen AD (2016) High-LET radiotherapy for adenoid cystic carcinoma of the head and neck: 15 years' experience with raster-scanned carbon ion therapy. Radiother Oncol 118: 272-80. [Crossref]

15. Jensen AD, Nikoghosyan AV, Poulakis M (2015) Combined intensity-modulated radiotherapy plus raster-scanned carbon ion boost for advanced adenoid cystic carcinoma of the head and neck results in superior locoregional control and overall survival. Cancer 121: 3001-3009. [Crossref]

Copyright: $\odot 2020$ Touimi SH. This is an open-access article distributed under the terms of the Creative Commons Attribution License, which permits unrestricted use, distribution, and reproduction in any medium, provided the original author and source are credited. 